\title{
Continuum double-exchange model
}

\author{
José María Román* and Joan Soto ${ }^{\dagger}$ \\ Department d'Estructura i Constituents de la Matèria and Institut de Física d'Altes Energies, Universitat de Barcelona, \\ Diagonal 647, E-08028 Barcelona, Catalonia, Spain
}

(Received 30 October 1998)

\begin{abstract}
We present a continuum model for doped manganites which consist of two species of quantum spin- $\frac{1}{2}$ fermions interacting with classical spin fields. The phase structure at zero temperature turns out to be considerably rich: antiferromagnetic insulator, antiferromagnetic two band conducting, canted two band conducting, canted one band conducting, and ferromagnetic one band conducting phases are identified, all of them being stable against phase separation. There are also regions in the phase diagram where phase separation occurs. [S0163-1829(99)07317-8]
\end{abstract}

\section{INTRODUCTION}

Doped manganites $\mathrm{La}_{1-x} A_{x} \mathrm{MnO}_{3}(A \text { divalent })^{1}$ are receiving quite a lot of both theoretical ${ }^{2-9}$ and experimental ${ }^{10}$ attention lately. These materials show an interesting interplay between magnetism and conductivity with intrincate phase diagrams which are still controversial.

In a cubic lattice the $3 d$ orbitals of Mn split into a $t_{2 g}$ triplet and an upper $e_{g}$ doublet. Due to the electronic repulsion and the Fermi statistics (Hund's rule) the three $t_{2 g}$ levels are always single occupied forming a core $S=\frac{3}{2}$ spin. The $e_{g}$ orbitals may be further split by a static Jahn-Teller distortion at small doping. ${ }^{11}$

The above features are encoded in the so-called double exchange models of different degrees of complexity. The simpler ones assume a strong Jahn-Teller distortion so that only the lower $e_{g}$ level is considered. Hence there is a single fermion field in each site, with a spin independent hopping term and a local interaction with the core spin. ${ }^{4,5}$ Core spins also interact among themselves with the usual Heisenberg term. Under certain assumptions ${ }^{12}$ the interaction with the core spin can be traded for an angle dependent hopping term. ${ }^{2,13}$ The next level of complexity consists of taking into account the two $e_{g}$ levels, ${ }^{6,7}$ and only very recently, the JahnTeller distortion has been incorporated dynamically by some authors. $^{9}$

It is the aim of this work to present a simple continuum model for doped manganites which also encodes the basic features above and, moreover, is exactly solvable for classical core spins. It produces a rich phase diagram which is in qualitative agreement with recent results and it shows, in addition, that stable canted phases exist. The main advantage with respect to previous approaches is that all the parameters of the material (lattice spacing, band curvature, Hund coupling, Heisenberg coupling, and doping) combine into only two constants. This allows us to present a two-dimensional phase diagram which holds for a large amount of materials.

\section{THE MODEL}

Cooperative phenomena are amenable of a fieldtheoretical description. When the phenomena do not depend on the details of the microscopic system but only on its long- wavelength behavior a continuum field-theory description is appropriated. The field-theoretical continuum model must contain the relevant degrees of freedom at long wavelengths, which depend on the particular systems and phenomena that are to be studied. In our case, these are doped manganites and their phase diagram at zero temperature. These systems are known to undergo a number of phase transitions when the doping is increased. They are insulating antiferromagnets (AFI) at zero doping and become conducting ferromagnets (FC) at large enough doping. What happens between these two regimes is still controversial, though most authors agree that the phase diagram is very rich and nontrivial. Early works on the subject suggested that an interesting intermediate conducting canted phase exists, ${ }^{13}$ but recent experimental ${ }^{10}$ and theoretical ${ }^{2,4}$ results indicate that the canted phase appears to be unstable against phase separation.

Theoretical work on the subject is based on variations of the double exchange models. The phase structure of the system is obtained from these models using certain simplifying assumptions (slave boson formalism, ${ }^{2}$ trial wave functions, ${ }^{4}$ etc.) or extensive numerical simulations, ${ }^{9}$ the scope of which is difficult to evaluate. We present below a continuum fieldtheoretical model which, as we shall argue, contains the relevant long-wavelength degrees of freedom of the system. Then our main assumption is going to be that the rich phase diagram of manganites can be understood from longwavelength physics only. As the model is exactly solvable, there are no further uncertainties due to uncontrolled approximations.

Since we wish our model to include the well established $\mathrm{AFI}$ and FC phases, we need at least an AF order-parameter field, a $\mathrm{F}$ order parameter field, and a I-C order parameter field. For the AF and $\mathrm{F}$ order parameter fields we shall use $\mathbf{M}_{1}(x)$ and $\mathbf{M}_{2}(x)$ the local magnetizations in the even and odd sublattices, respectively. Both in the AF and $\mathrm{F}$ phases these local magnetizations are smoothly varying fields. In the AF phase $\mathbf{M}_{1}(x) \mathbf{M}_{2}(x) \sim-1$ whereas in the $\mathrm{F}$ phase $\mathbf{M}_{1}(x) \mathbf{M}_{2}(x) \sim 1$. For the I-C order parameter one could think of introducing a single slowly varying spin- $\frac{1}{2}$ fermion field together with a chemical potential which regulates the doping. When the chemical potential is below the energy gap of the lowest spin state we have an I phase, when it overtakes this energy gap we have a one band $\mathrm{C}$ phase, and when it 
overtakes the energy gap of the highest spin state we have a two band $\mathrm{C}$ phase. However, a spin- $\frac{1}{2}$ field naturally couples to the local magnetization, which changes abruptly from the even to the odd sublattice in the AF phase. Hence in this phase a single spin- $\frac{1}{2}$ field cannot be slowly varying over the system. We need at least two slowly varying spin- $\frac{1}{2}$ fermionic fields, $\psi_{1}(x)$ which couples to the magnetization in the even sublattice $\mathbf{M}_{1}(x)$ and $\psi_{2}(x)$ which couples to the magnetization in the odd sublattice $\mathbf{M}_{2}(x)$. Since the conductivity is due to fermions moving from one sublattice to the other one a (spin independent) hopping term is introduced. The allowed values of the chemical potential will be limited by the physical condition that no conduction must exist when the hopping parameter vanishes.

The model must be $S U(2)$ spin invariant since the magnetic interactions emerge from the usual superexchange mechanism together with the Hund's rule. The space-time symmetries of the underlying crystal must also be implemented and will be the only remains of the microscopic lattice structure. For simplicity we shall take a cubic lattice and comment later on the slight modifications that occur for other crystals.

The Lagrangian of the model reads

$$
\begin{aligned}
\mathcal{L}(x)= & \psi_{1}^{\dagger}(x)\left((1+i \boldsymbol{\epsilon}) i \partial_{0}+\frac{\partial_{i}^{2}}{2 m}+\mu+J_{H} \frac{\boldsymbol{\sigma}}{2} \mathbf{M}_{1}(x)\right) \psi_{1}(x) \\
& +\psi_{2}^{\dagger}(x)\left((1+i \epsilon) i \partial_{0}+\frac{\partial_{i}^{2}}{2 m}+\mu+J_{H} \frac{\boldsymbol{\sigma}}{2} \mathbf{M}_{2}(x)\right) \psi_{2}(x) \\
& +t\left[\psi_{1}^{\dagger}(x) \psi_{2}(x)+\psi_{2}^{\dagger}(x) \psi_{2}(x)\right]-J_{\mathrm{AF}} \mathbf{M}_{1}(x) \mathbf{M}_{2}(x) .
\end{aligned}
$$

The size of the parameters in the model is estimated by comparing them with the naive continuum limit of lattice double exchange models. For a cubic lattice we have $2 m$ $\sim 1 / a^{2} t^{l}, t \sim z t^{l}, J_{H} \sim J_{H}^{l}$, and $J_{\mathrm{AF}} \sim z J_{\mathrm{AF}}^{l} / a^{3}>0$, where $a$ is the lattice spacing, $z=6$ is the coordination number, and the superscript $l$ means the analogous lattice quantity. The fields $\psi_{i}(x)$ may describe either electrons or holes. Since the conduction in actual doped manganites is due to holes, one should better figure out $\psi_{i}(x)$ as hole annihilating fields. Recall that for holes $J_{H}$ is negative whereas it is positive for electrons. This sign however is going to be irrelevant as far as the phase diagram is concerned.

The Lagrangian above is invariant under the following transformations:

(i) global $S U(2)$ spin transformations:

$$
\begin{aligned}
\psi_{i}(x) & \rightarrow g \psi_{i}(x) \\
M_{i}^{a}(x) & \rightarrow R_{b}^{a} M_{i}^{b}(x) \quad(i=1,2),
\end{aligned}
$$

(ii) primitive translations:

$$
\begin{array}{cc}
\psi_{1}(x) \rightarrow \psi_{2}(x), & \psi_{2}(x) \rightarrow \psi_{1}(x), \\
\mathbf{M}_{1}(x) \rightarrow \mathbf{M}_{2}(x), & \mathbf{M}_{2}(x) \rightarrow \mathbf{M}_{1}(x),
\end{array}
$$

(iii) point group transformations, given by the group $m \overline{3} m$ :

$$
\begin{gathered}
\psi_{i}(x) \rightarrow g_{\xi} \psi_{i}\left(\xi^{-1} x\right) \\
M_{i}^{a}(x) \rightarrow R_{b}^{a}(\xi) M_{i}^{b}\left(\xi^{-1} x\right) \quad(i=1,2),
\end{gathered}
$$

when the point group transformation $\xi$ maps points in the same sublattice, and

$$
\begin{array}{cc}
\psi_{1}(x) \rightarrow g_{\xi} \psi_{2}\left(\xi^{-} x\right), & \psi_{2}(x) \rightarrow g_{\xi} \psi_{1}\left(\xi^{-1} x\right), \\
M_{1}^{a}(x) \rightarrow R_{b}^{a}(\xi) M_{2}^{b}\left(\xi^{-1} x\right), & M_{2}^{a}(x) \rightarrow R_{b}^{a}(\xi) M_{1}^{b}\left(\xi^{-1} x\right),
\end{array}
$$

when the transformation $\xi$ maps points of different sublattices. Anyway, the rotations $g_{\xi}$ and $R_{b}^{a}(\xi)$ can be absorbed by a $S U(2)$ transformation and the change of sublattice in Eq. (2.5) by a primitive translation. Hence, in practice, we only have to care about the transformation of the coordinates.

(iv) Time reversal,

$$
\begin{gathered}
\psi_{i}(x) \rightarrow C \psi_{i}^{*}(T x) \\
\mathbf{M}_{i}(x) \rightarrow-\mathbf{M}_{i}(T x)
\end{gathered} \quad C=e^{-i \pi \sigma^{2} / 2}=-i \sigma^{2}, \quad(i=1,2),
$$

where $T x=(-t, \mathbf{x})$.

\section{EFFECTIVE POTENTIAL}

In order to find out how the ground state of the system changes as a function of the chemical potential, we shall calculate the effective potential and minimize it with respect to the order parameters $\mathbf{M}_{1}$ and $\mathbf{M}_{2}$. We shall assume that the ground-state configuration corresponds to constant magnetizations both in the odd and even sublattices. Hence the effective potential is to be minimized with respect to the angle $\theta$ between $\mathbf{M}_{1}$ and $\mathbf{M}_{2}$ only. We use $y=\cos (\theta / 2)$. When $y=0,0<y<1$, and $y=1$ we have an antiferromagnetic, canted, and ferromagnetic phase, respectively.

The effective potential is obtained by integrating out the fermion fields in the path integral, and it is formally given by

$$
V_{\text {eff }}=J_{\mathrm{AF}} \mathbf{M}_{1} \mathbf{M}_{2}+i t r \log \hat{O} / V T,
$$

where

$$
\hat{O}=\left(\begin{array}{cc}
(1+i \epsilon) i \partial_{0}+\partial_{i}^{2} / 2 m+\mu+\frac{J_{H}}{2} \boldsymbol{\sigma} \mathbf{M}_{1} & t \\
t & (1+i \epsilon) i \partial_{0}+\partial_{i}^{2} / 2 m+\mu+\frac{J_{H}}{2} \boldsymbol{\sigma} \mathbf{M}_{2}
\end{array}\right)
$$


and the trace is both on spin indices and space-time coordinates. VT is the volume of the space time.

If $\hat{O}$ has eigenvalues $\lambda_{n}$,

$$
\operatorname{tr} \log \hat{O}=\sum_{n} \log \lambda_{n} .
$$

We have then to diagonalize the operator $\hat{O}$. Since it contains only constant fields the diagonalization with respect to the space time is trivially attained by plane waves. The diagonalization with respect to the spin indices is a simple linear algebra problem. We obtain

$$
\begin{aligned}
& \lambda_{n}=O_{i}(q)=(1+i \epsilon) \omega-\frac{\mathbf{k}^{2}}{2 m}-\Omega_{i}, \\
& \Omega_{i}= \pm \frac{\left|J_{H}\right| M}{2} \sqrt{1+\gamma^{2} \pm 2 \gamma \cos \frac{\theta}{2}}-\mu, \quad \gamma \equiv \frac{2 t}{\left|J_{H}\right| M} .
\end{aligned}
$$

$q=(\omega, \mathbf{k})$ and $M=\left|\mathbf{M}_{1}\right|=\left|\mathbf{M}_{2}\right|=\frac{3}{2}$. The restriction for the values of the chemical potential in the model implies that at most the two lower eigenvalues in Eq. (3.5) may contribute. This motivates the following reparametrization of the chemical potential:

$$
\mu=-\frac{\left|J_{H}\right| M}{2} \sqrt{1+\gamma^{2}-2 \gamma y_{0}} \quad\left(-1<y_{0}<y_{0}^{\max }=\gamma / 2\right),
$$

which eases comparison with the energy levels in Eq. (3.5) $[y=\cos (\theta / 2)]$. In order to simplify the analysis we assume $\gamma$ small and keep only linear terms in $\gamma$ in the relevant eigenvalues above. Namely,

$$
\Omega_{i}=-\frac{\left|J_{H}\right| M}{2} \gamma\left(y_{0} \pm y\right) .
$$

This is justified for $t \ll J_{H}$, as it turns out to be the case for the actual materials. ${ }^{14}$ Anyway, this simplification can be lifted with the only drawback that the few analytic expressions below must also be substituted by numerical analysis.

In order to calculate the sum (3.3) we have used $\zeta$-function techniques, ${ }^{15}$ which are explained in the appendix. We obtain the effective potential (for $\mu<0$ )

$$
\begin{aligned}
V_{\text {eff }}= & V_{0}\left\{\left(2 y^{2}-1\right)-A\left[\left(y_{0}+y\right)^{5 / 2} \theta\left(y_{0}+y\right)\right.\right. \\
& \left.\left.+\left(y_{0}-y\right)^{5 / 2} \theta\left(y_{0}-y\right)\right]\right\},
\end{aligned}
$$

where we have defined

$$
V_{0}=J_{\mathrm{AF}} M^{2}, \quad A=\frac{(2 m)^{3 / 2} t^{5 / 2}}{15 \pi^{2} J_{\mathrm{AF}} M^{2}}=\frac{z^{3 / 2}}{15 \pi^{2}} \frac{t}{\left(J_{\mathrm{AF}} a^{3} M^{2}\right)} .
$$

\section{PHASE STRUCTURE}

The possible phases of the model are obtained by minimizing Eq. (3.8) with respect to $y$ for the different values of the parameters $A$ and $y_{0}$. The number of conducting bands is given by the number of $\theta$ functions in Eq. (3.8) which contribute to the effective potential at the minimum.

In order to gain some qualitative understanding and to make the minimization procedure systematic we shall first separate the cases $y_{0}<0$ and $y_{0}>0$. For each case we shall work out the stability conditions for $\operatorname{AF}(y=0)$, canted $(0$ $<y<1)$, and $\mathrm{F}(x=1)$ phases. After that we shall compare the energy of the stable phases and obtain the curves which separate them.

The stability conditions are given for the different phases by

$$
\begin{array}{ll}
\text { AF: } & V_{\mathrm{eff}}^{\prime}(0)>0 \quad \text { or } \quad V_{\mathrm{eff}}^{\prime}(0)=0 \quad V_{\mathrm{eff}}^{\prime \prime}(0)>0, \\
\text { C: } \quad V_{\mathrm{eff}}^{\prime}\left(y_{c}\right)=0 \quad V_{\mathrm{eff}}^{\prime \prime}\left(y_{c}\right)>0, \\
\text { F: } \quad V_{\mathrm{eff}}^{\prime}(1)<0 .
\end{array}
$$

Let us then consider first the case $y_{0}<0$. Clearly for $y_{0}$ $<-1$ the unique existing phase is the AFI phase. In the case $-1<y_{0}<0$ only the lowest of the four spin eigenvalues may contribute to the effective potential. The stability conditions yield the following stable phases:

$$
\begin{aligned}
& \text { AFI: } \quad y=0, \\
& \text { FC: } y=1, \quad A\left(1+y_{0}\right)^{3 / 2}>\frac{8}{5} .
\end{aligned}
$$

The canted phase is not stable as it can be seen from the condition $V_{\text {eff }}^{\prime}\left(y_{c}\right)=0$,

$$
y_{c}=\frac{5}{8} A\left(y_{0}+y_{c}\right)^{3 / 2},
$$

which has at most one solution $y_{c} \in\left[-y_{0}, 1\right]$. Since $V_{\text {eff }}$ is continuous, and increasing at $y=0$ this solution must be a maximum when it exists.

The curve $V_{\text {eff }}(0)=V_{\text {eff }}(1)$ in the plain $\left(y_{0}, A\right)$, which separates the $\mathrm{AF}$ and $\mathrm{F}$ phases, reads

$$
A\left(1+y_{0}\right)^{5 / 2}=2, \quad\left(-1<y_{0}<0\right) .
$$

Above this curve the $\mathrm{F}$ phase is favored against the AF phase and vice versa.

Consider next the case $0<y_{0}<1$. The stability conditions are given by

AFC2: $\quad y=0, \quad A y_{0}^{1 / 2}<\frac{8}{15}$,

CC2: $\quad 5 A\left(y_{c}^{2}+3 y_{0}^{2}\right) / 4=\left(y_{0}+y_{c}\right)^{3 / 2}+\left(y_{0}-y_{c}\right)^{3 / 2}, \quad 8 / 15<A y_{0}^{1 / 2}<2 \sqrt{2} / 5$,

$\mathrm{CC} 1: \quad y_{c}=5 A\left(y_{0}+y_{c}\right)^{3 / 2} / 8, \quad A y_{0}^{1 / 2}>2 \sqrt{2} / 5$,

FC1: $y=1, \quad A\left(1+y_{0}\right)^{3 / 2}>\frac{8}{5}$, 
where AFC2, CC2, CC1, and FC1 stand for antiferromagnetic two band conducting, canted two band conducting, canted one band conducting, and ferromagnetic one band conducting, respectively. Notice that AF and canted phases do not compete among them, but only with the $\mathrm{F}$ phase. The curves providing the boundary between the different phases are given by

$$
\begin{array}{ll}
\mathrm{AFC} 2-\mathrm{FC} 1: & A\left[\left(1+y_{0}\right)^{5 / 2}-2 y_{0}^{5 / 2}\right]=2, \quad 0<y_{0}<0.127195, \\
\mathrm{AFC} 2-\mathrm{CC} 2: & A y_{0}^{1 / 2}=\frac{8}{15}, \quad 0.127195<y_{0}<1, \\
\mathrm{CC} 2-\mathrm{FC} 1: & 5 A\left(y_{2}^{2}+3 y_{0}^{2}\right) / 4=\left(y_{0}+y_{2}\right)^{3 / 2}+\left(y_{0}-y_{2}\right)^{3 / 2}, \quad 0.127195<y_{0}<0.168457, \\
\mathrm{CC} 2-\mathrm{CC} 1: & A y_{0}^{1 / 2}=2 \sqrt{2} / 5, \quad 0.168457<y_{0}<1, \\
\mathrm{CC} 1-\mathrm{FC} 1: & 5 A\left(y_{0}+y_{1}\right)^{3 / 2} / 8=y_{1}, \quad 0.168457<y_{0}<0.5, \\
\mathrm{CC} 1-\mathrm{FC} 1: & 5 A\left(1+y_{0}\right)^{3 / 2} / 8=1, \quad 0.5<y_{0}<1,
\end{array}
$$

where $y_{1}$ and $y_{2}$ are given implicitly by the equations

$$
\begin{aligned}
& \quad\left[\left(1+y_{0}\right)^{5 / 2}-\left(y_{0}+y_{2}\right)^{5 / 2}-\left(y_{0}-y_{2}\right)^{5 / 2}\right]\left[\left(y_{0}+y_{2}\right)^{3 / 2}\right. \\
& \left.\quad+\left(y_{0}-y_{2}\right)^{3 / 2}\right]=\frac{5}{2}\left(1-y_{2}^{2}\right)\left(y_{2}^{2}+3 y_{0}^{2}\right) \\
& \left(y_{1}+y_{0}\right)^{5 / 2}+2\left(1+y_{0}\right)^{1 / 2}\left(y_{1}+y_{0}\right)^{2} \\
& +3\left(1-y_{0}\right)\left(y_{1}+y_{0}\right)^{3 / 2}+4\left(1-2 y_{0}\right)\left(1+y_{0}\right)^{1 / 2}\left(y_{1}+y_{0}\right) \\
& -8 y_{0}\left(1+y_{0}\right)\left(y_{1}+y_{0}\right)^{1 / 2}-4 y_{0}\left(1+y_{0}\right)^{3 / 2}=0 .
\end{aligned}
$$

The outcome is plotted in Fig. 1.

Recall that Fig. 1 actually does not plot a phase diagram against doping but against $y_{0}$ which is related to the chemical potential rather than to the number of conducting fermions or doping. Recall also that $V_{\text {eff }}$ is to be regarded as a (zero temperature) grand canonical potential rather than as a free energy. The doping is introduced via

$$
x=-a^{3} \frac{\partial V_{\mathrm{eff}}}{\partial \mu}=-\frac{a^{3}}{t} \frac{\partial V_{\mathrm{eff}}}{\partial y_{0}}
$$

provided that one molecule exists per unit cell with a lattice parameter $a$. Taking into account Eq. (3.9) the doping corresponding to the different phases reads

$$
\begin{aligned}
\mathrm{AFI}: & x=0, \\
\mathrm{AFC} 2: & x=\frac{z^{3 / 2}}{6 \pi^{2}} 2 y_{0}^{3 / 2}, \\
\mathrm{CC} 2: \quad x & =\frac{z^{3 / 2}}{6 \pi^{2}}\left[\left(y_{0}+y_{c}\right)^{3 / 2}+\left(y_{0}-y_{c}\right)^{3 / 2}\right], \\
\mathrm{CC} 1: & x=\frac{z^{3 / 2}}{6 \pi^{2}}\left(y_{0}+y_{c}\right)^{3 / 2}, \\
\mathrm{FC} 1: & x=\frac{z^{3 / 2}}{6 \pi^{2}}\left(1+y_{0}\right)^{3 / 2},
\end{aligned}
$$

where the $y_{c}$ for the $\mathrm{CC} 2$ and $\mathrm{CC} 1$ phases are given in Eq. (4.5).
These expressions for the doping permit us to establish that all our phases are thermodynamically stable, unlike the ones observed in Refs. 3 and 4 . This is easily proven from the stability condition $\partial \mu / \partial x>0$. For the $\mathrm{F}$ and AF phases this is trivially obtained, whereas canted phases are stable if they are below the curves:

$$
\begin{aligned}
\mathrm{CC} 2: & 5 A y / 3=\left(y_{0}+y\right)^{1 / 2} \\
& -\left(y_{0}-y\right)^{1 / 2} y^{2}-5 y_{0}^{2}+4 y_{0}\left(y_{0}^{2}-y^{2}\right)^{1 / 2}=0, \quad\left(y<y_{0}\right) \\
\mathrm{CC} 1: & A y_{0}^{1 / 2}=\frac{16}{15} \sqrt{3} .
\end{aligned}
$$

This is always the case as it is shown in Fig. 1 where we have plotted the two curves.

Once we have the expressions (4.9) for the doping it is straightforward to translate Fig. 1 to a more conventional

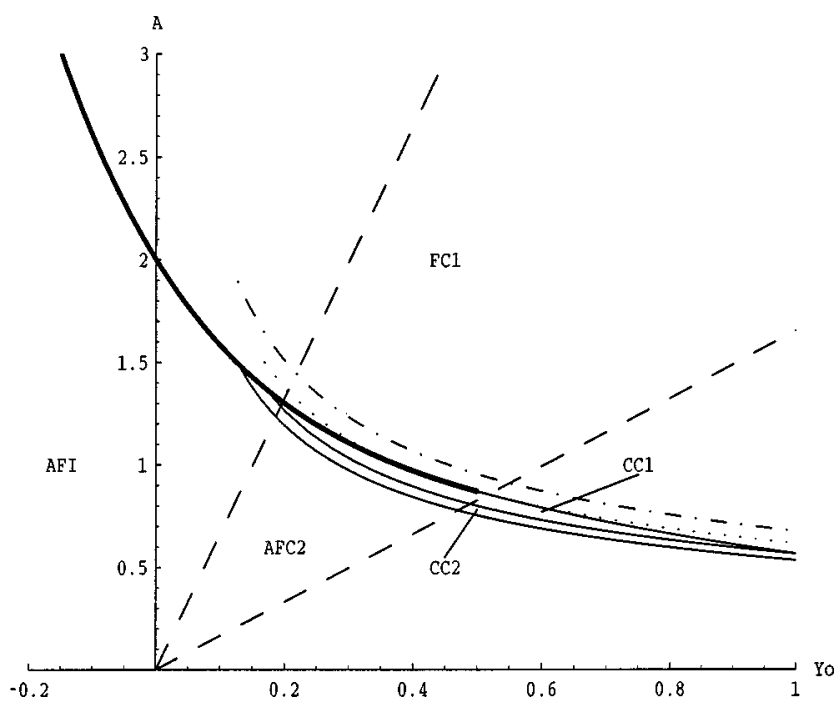

FIG. 1. Phase diagram in the $\left(y_{0}, A\right)$ plane. The thick solid line corresponds to first-order transitions whereas the remaining solid lines to second-order ones. The dotted and dashed-dotted lines are the upper stability boundaries for the $\mathrm{CC} 1$ and $\mathrm{CC} 2$ phases, respectively. The two dashed lines are the boundaries for the reliability of our model for $z\left|J_{H}\right| M / 2\left(J_{\mathrm{AF}} a^{3} M^{2}\right) \sim 50$ and $z\left|J_{H}\right| M / 2\left(J_{\mathrm{AF}} a^{3} M^{2}\right)$ $\sim 200$, respectively. Only the part of the phase diagram to the left of the corresponding dashed line is trustworthy in each case. 


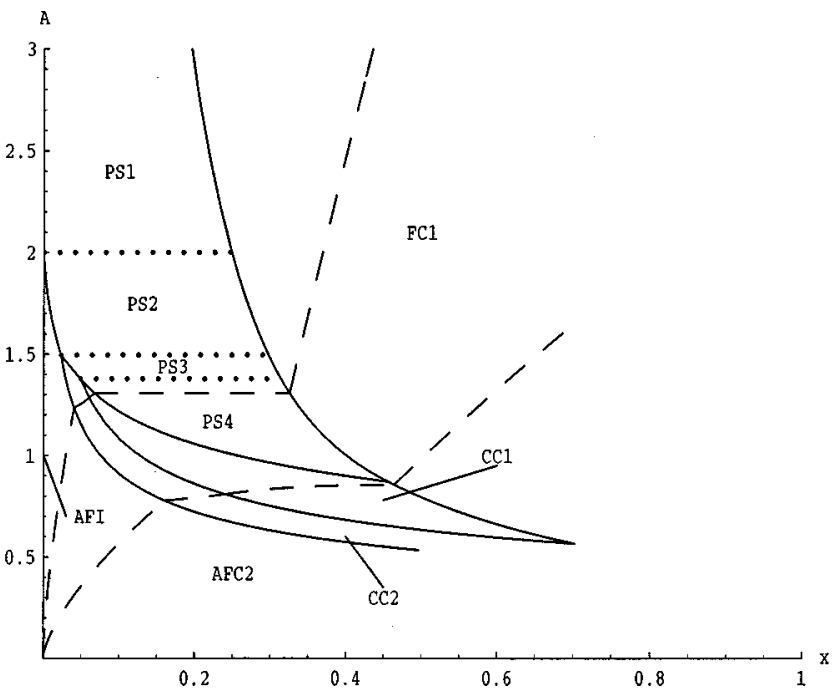

FIG. 2. Phase diagram in the $(x, A)$ plane. PS $i(i=1,2,3,4)$ indicates the new regions where the phases at their boundary may coexist. The $x=0$ axis corresponds to the AFI phase. The two dashed lines are the boundaries for the reliability of our model for $z\left|J_{H}\right| M / 2\left(J_{\mathrm{AF}} a^{3} M^{2}\right) \sim 50$ and $z\left|J_{H}\right| M / 2\left(J_{\mathrm{AF}} a^{3} M^{2}\right) \sim 200$, respectively. Only the part of the phase diagram to the left of the corresponding dashed line is trustworthy in each case.

phase diagram where the doping $x$ appears in one of the axes. This is given in Fig. 2 (recall $z=6$ ).

It is interesting to notice that in Fig. 2 new regions arise, which we have denoted PSi $(i=1,2,3,4)$, between the FC1 and the $\mathrm{AFI}, \mathrm{AFC} 2, \mathrm{CC} 2$, and $\mathrm{CC} 1$ phases, respectively. This is due to the fact that the thick solid line separating FC1 and $\mathrm{AFI}, \mathrm{AFC} 2, \mathrm{CC} 2$, and $\mathrm{CC} 1$ in Fig. 1 corresponds to a first-order phase transition. Along this line two stable inequivalent minima have the same energy and the chemical potential cannot be traded by the doping. These regions are likely to consist of coexisting domains where the two phases at the boundary are realized (phase separation). ${ }^{9}$ AFI and FC1 would coexist in PS1, as it has been observed in recent works. ${ }^{3,4} \mathrm{FC} 1$ and $\mathrm{AFC} 2, \mathrm{CC} 2$, and $\mathrm{CC} 1$ would coexist in PS1, PS2, and PS3, respectively.

As mentioned in Sec. III, the fact that for $t=0$ we do not permit conductivity restricts the values that the chemical potential takes to $y_{0}<y_{0}^{\max }=\gamma / 2$. By substituting this expression in $A$ we obtain

$$
A=\frac{2 z^{1 / 2}}{15 \pi^{2}} \frac{z\left|J_{H}\right| M}{2\left(J_{\mathrm{AF}} a^{3} M^{2}\right)} y_{0}^{\max },
$$

which gives the boundary of validity for our results. It turns out to be a straight line in Fig. 1 provided that $J_{\mathrm{AF}}$ and $J_{H}$ remains constant as $y_{0}^{\max }$ moves, which can be straightforwardly translated to Fig. 2. Only the phase diagram to the left of this curve is trustworthy.

We take for the coupling constants $t /\left(J_{\mathrm{AF}} a^{3} M^{2}\right)$ $\sim 10-20$ and $z\left|J_{H}\right| M / 2\left(J_{\mathrm{AF}} a^{3} M^{2}\right) \sim 50-200$, which is compatible with the values given in the literature. For these values $A \sim 1-2$, and the two extreme validity curves are displayed as dashed lines in Figs. 1 and 2.

\section{CONCLUSIONS}

We have presented a simple model in the continuum which is able to describe the rich phase structure of doped manganites for a wide range of these materials. We have assumed an underlying cubic crystal for simplicity. Nevertheless, the orthorhombic distortion can be easily accommodated by the following simple changes in the physical parameters: $m^{3} \rightarrow m_{x} m_{y} m_{z}, a^{3} \rightarrow a b c, \quad J_{\mathrm{AF}} \rightarrow J_{x}+J_{y}+J_{z}$, and $t$ $\rightarrow t_{x}+t_{y}+t_{z}$. In practice this does not modify our results since it would only lead to a different $A$, which is anyway a free parameter in our phase diagrams. This fact also suggests that the structural transitions that these materials undergo when increasing the doping ${ }^{14}$ are not essential in order to understand the F-AF and I-C transitions.

An important feature of our results is that the two canted phases that we observe are stable against phase separation, unlike in some previous works. ${ }^{3,4} \mathrm{We}$ also observe regions in the phase diagram where phase separations of several kinds may occur. If we plug realistic values for the physical parameters we find $A \sim 1-2$. Within this range the following sequences of phases are possible upon increasing $x$ : (i) AFIPSI-FC1, (ii) AFI-AFC2-PS2-FC1, (iii) AFI-AFC2-CC2PS3-FC1, (iv) AFI-AFC2-CC2-CC1-PS4-FC1. Recall also that in PS3 and PS4 ferromagnetic and canted phases coexist. This may explain some controversial results obtained by different authors.

Let us also mention that the two fermion fields $\psi_{1}(x)$ and $\psi_{2}(x)$ accommodate the $e_{g}$ doublet in our model. Indeed in the AF phase the two lower and two higher eigenvalues (3.4) are degenerated. In the $\mathrm{F}$ and $\mathrm{C}$ phases the degeneracy is lifted. This implies that the splitting between the two $e_{g}$ levels receives a contribution from the dynamics of the conducting fermions in addition to that from the static Jahn-Teller distortion.

The model can be used in the future to study the temperature dependence of the phase diagram. Fluctuations due to spin waves in all the phases (including the canted ones) can also be incorporated. ${ }^{16}$ It would also be interesting to see if the model can be generalized to accommodate the JahnTeller distortion dynamically.

\section{ACKNOWLEDGMENTS}

We are indebted to F. Guinea and L. Brey for introducing us to the physics of manganites, and to J. González for an illuminating discussion in the early stages of this work. J.M.R. was supported by a Basque Government F.P.I. grant. Financial support from CICYT, Contract No. AEN95-0590 and from CIRIT, Contract No. GRQ93-1047 is also acknowledged.

\section{APPENDIX: $\zeta$-FUNCTION TECHNIQUES}

The $\zeta$-function techniques provide a very efficient way to calculate the trace of the logarithm of operators. ${ }^{15}$ The $\zeta$ function associated to an operator $\hat{O}$ is defined as

$$
\zeta \hat{O}(s):=\operatorname{tr} \hat{O}^{-s}=\sum_{n} \lambda_{n}^{-s}
$$

Then 


$$
\sum_{n} \log \lambda_{n}=-\left.\frac{d}{d s} \zeta \hat{o}^{(s)}\right|_{s=0} .
$$

Consider the operator $\hat{O}$ in Eq. (3.2). Once the spin diagonalization is performed we only have to consider the space-time trace over a generic spin eigenvalue denoted by $\hat{O}_{i}$. Since the real part of the operator $-i \hat{O}_{i}$ is positive for positive energies and negative for negative ones, due to the term $i \epsilon \omega$ in Eq. (3.4), it is convenient to consider the integral form of $\zeta_{\hat{O}}(s)$ over positive and negative energies of separately:

$$
\begin{aligned}
\operatorname{tr}\left[\left(\hat{O}_{i} \theta(-\omega)\right)^{-s}\right]= & \frac{(-i)^{-s}}{\Gamma(s)} \int_{0}^{\infty} d \tau \tau^{s-1} \int_{-\infty}^{0} \frac{d \omega}{2 \pi} \frac{d^{3} \mathbf{k}}{(2 \pi)^{3}} \\
& \times e^{-i O_{i}(q) \tau} V T, \\
\operatorname{tr}\left[\left(\hat{O}_{i} \theta(\omega)\right)^{-s}\right]= & \frac{i^{-s}}{\Gamma(s)} \int_{0}^{\infty} d \tau \tau^{s-1} \int_{0}^{\infty} \frac{d \omega}{2 \pi} \frac{d^{3} \mathbf{k}}{(2 \pi)^{3}} \\
& \times e^{i O_{i}(q) \tau} V T .
\end{aligned}
$$

After the energy and momentum integration we obtain the expressions

$$
\begin{aligned}
\operatorname{tr}\left[\left(\hat{O}_{i} \theta(-\omega)\right)^{-s}\right]= & \frac{V T}{16 \pi}\left(\frac{2 m}{\pi}\right)^{3 / 2} \\
& \times \frac{\Gamma(s-5 / 2)}{\Gamma(s)}(-i)^{-s-5 / 2}\left(-i \Omega_{i}\right)^{s+5 / 2}, \\
\operatorname{tr}\left[\left(\hat{O}_{i} \theta(\omega)\right)^{-s}\right]= & -\frac{V T}{16 \pi}\left(\frac{2 m}{\pi}\right)^{3 / 2} \\
& \times \frac{\Gamma(s-5 / 2)}{\Gamma(s)}(-i)^{s+5 / 2}\left(i \Omega_{i}\right)^{-s+5 / 2} .
\end{aligned}
$$

$(\mathrm{A} 4 \mathrm{~b})$
We need the derivative of the above with respect to $s$ at $s$ $=0$. The presence of $1 / \Gamma(s) \sim s$ makes the evaluation very easy, giving rise to

$$
\begin{aligned}
-\left.\frac{d}{d s} \zeta_{\hat{O}}(s)\right|_{s=0}= & \frac{V T(2 m)^{3 / 2}}{30 \pi^{2}}\left[i^{5 / 2}\left(-i \Omega_{i}\right)^{5 / 2}\right. \\
& \left.-(-i)^{5 / 2}\left(i \Omega_{i}\right)^{5 / 2}\right] .
\end{aligned}
$$

The expression between square brackets vanishes when $\Omega_{i}>0$, i.e., when the chemical potential is bellow the energy of the $i$ th state, and is nonzero when $\Omega_{i}<0$, i.e., when the chemical potential is above the energy of the $i$ th state. This leads to the effective potential (for $\mu<0, y_{0}<y_{0}^{\max }<1$ )

$$
\begin{aligned}
V_{\mathrm{eff}}= & V_{0}\left\{\left(2 y^{2}-1\right)-\frac{A}{\gamma^{5 / 2}}\right. \\
& \times\left[\left(\sqrt{1+\frac{2 \gamma y}{1+\gamma^{2}}}-\sqrt{1-\frac{2 \gamma y_{0}}{1+\gamma^{2}}}\right)^{5 / 2} \theta\left(y_{0}+y\right)\right. \\
& \left.\left.+\left(\sqrt{1-\frac{2 \gamma y}{1+\gamma^{2}}}-\sqrt{1-\frac{2 \gamma y_{0}}{1+\gamma^{2}}}\right)^{5 / 2} \theta\left(y_{0}-y\right)\right]\right\},
\end{aligned}
$$

where $y=\cos (\theta / 2)$, whereas $\gamma, y_{0}$, and $V_{0}$ are defined in Eqs. (3.5), (3.6), and (3.9), respectively.

$$
A=\frac{(2 m)^{3 / 2}}{15 \pi^{2} J_{\mathrm{AF}} M^{2}}\left(t \sqrt{1+\gamma^{2}}\right)^{5 / 2} .
$$

Equation (3.7) follows from the above by keeping only terms linear in $\gamma$.
*Electronic address: roman@physics.vivc.edu, present address: Loomis Laboratory of Physics, 1110 West Green St., Urbana, IL 61801-3080.

†Electronic address: soto@ecm.ub.es

${ }^{1}$ G. H. Jonker and J. H. Van Santen, Physica (Amsterdam) 50, 337 (1950).

${ }^{2}$ D. P. Arovas and F. Guinea, Phys. Rev. B 58, 9150 (1998).

${ }^{3}$ D. P. Arovas, G. Gómez-Santos, and F. Guinea, cond-mat/9805399, Phys. Rev. B (to be published, 1 June 1999).

${ }^{4}$ M. Yu Kagan, D. I. Khomskii, and M. Mostovoy, cond-mat/9804213 (unpublished).

${ }^{5}$ L.-J. Zou, Q.-Q. Zheng, and H. Q. Liu, Phys. Rev. B 56, 13669 (1997).

${ }^{6}$ A. J. Millis, P. B. Littlewood, and B. I. Shraiman, Phys. Rev. Lett. 75, 5144 (1995).

${ }^{7}$ R. Maezono, S. Ishihara, and N. Nagaosa, Phys. Rev. B 58, 11583 (1998).
${ }^{8}$ D. I. Golosov, M. R. Norman, and K. Levin, Phys. Rev. B 58, 8617 (1998).

${ }^{9}$ S. Yunoki, A. Moreo, and E. Dagotto, Phys. Rev. Lett. 81, 5612 (1998); E. Dagotto, S. Yunoki, and A. Moreo, cond-mat/9809380 (unpublished).

${ }^{10}$ J. W. Lynn et al., Phys. Rev. Lett. 76, 4046 (1996); Y. Yamada et al., ibid. 77, 904 (1996); G. Allodi et al., Phys. Rev. B 56, 6036 (1997); J. M. De Teresa et al., ibid. 57, 3305 (1998); M. Hennion et al., Phys. Rev. Lett. 81, 1957 (1998); Wei Bao et al., Solid State Commun. 98, 55 (1996).

${ }^{11}$ C. Zener, Phys. Rev. 82, 403 (1951).

${ }^{12}$ P. W. Anderson and H. Hasegawa, Phys. Rev. 100, 675 (1955).

${ }^{13}$ P. G. De Gennes, Phys. Rev. 118, 141 (1960).

${ }^{14}$ A. P. Ramirez, J. Phys.: Condens. Matter 9, 8171 (1997).

${ }^{15}$ E. Elizalde et al., Zeta Regularization Techniques with Applications (World Scientific, Singapore, 1994).

${ }^{16}$ J. M. Román and J. Soto (unpublished) 